\title{
Structural study of Topoisomerase IV-DNA-Levofloxacin complexes from Streptococcus pneumoniae
}

\author{
Xilan Wang ${ }^{1}$ \\ ${ }^{1}$ King's College London, Faculty of Life Sciences and Medicine, SE1 IUL, UK
}

\begin{abstract}
S. pneumoniae is an important pathogen causing pulmonary infection, acute otitis media and purulent meningitis in infants and children. Type II topoisomerases are enzymes that play essential roles in DNA replication, chromosome segregation and recombination throughout all living organisms. Topoisomerases IV can make a transient break in DNA strands in one chromosome. These enzymes are very important antibacterial as well as anticancer targets and potential anti-trypanosomal targets. Levofloxacin has shown efficient inhibition of Type II topoisomerases in S. pneumoniae. Its mechanism of action is to inhibit the activity of DNA topoisomerase, prevent bacterial DNA synthesis and replication leading to bacterial death. We focused on solving the key structures of topoisomerase IV-DNA-levofloxacin complexes by negative staining electron microscopy and the resulting model was obtained at $32 \AA$ by $3 \mathrm{D}$ autorefine in Relion 3.0. This study was to try and obtain the structure of the whole complex with DNA bound in the G-gate and the T-gate in order to study DNA capture and transport in type II topoisomerases.
\end{abstract}

\section{Induction}

The topological structure of DNA is based on the double helix, which further distorts the specific spatial structure. The supercoiling structure is the main form of the topological structure. Huang proved that topoisomerase II might be associated with the formation of the superagglutinative structure of species [1]. As a genetic material, the diversity of DNA topological structure is not only the basis of biodiversity, but also essential in biological processes.

DNA topoisomerases are enzymes that can control and regulate the topological state of DNA [2]. DNA topoisomerases exists widely in various biological species and its structure and function have been widely concerned.

The mechanism of DNA topoisomerase is to break and connect the phosphodiester bonds of DNA strands by two consecutive transesterification reactions, mediate the instantaneous break and reconnection of single or double strands of DNA and change the topological structure of DNA [3]. Type II topoisomerase is mainly divided into two types, one is DNA gyrase and the other is topoisomerase IV [4].

The main structure of Topoisomerase IV is composed of two $\mathrm{C}$ subunits (ParC gene coding) and two $\mathrm{E}$ subunits (ParE gene coding), and unlinks catenated daughter chromosomes during chromosomal segregation through ATP hydrolysis. As for ParE, it has two regions, one is the ATPase domain and the other is the TOPRIM domain. ATP molecules bind to ATPase domain to capture T-DNA by dimerization. The ParC region contains a $\mathrm{N}$ terminal binding with a G-DNA, tower, and
C-gate. The N-terminal of the ParC region is CAPshaped and because it contains the helix-turn-helix region, which is also known as the Winged Helix Domain (WHD). The breakage-reunion domain is called C-gate containing two long a-helix which produces $30 \AA$ hole. G-DNA binds with The WHD region of ParC and with the TOPRIM domain of ParE to form a U-shaped structure. In the core region of DNA-gates, active residues tyr118 on parC is linked to the $5^{\prime}$ phosphoric acid end of DNA and there is a $4 \mathrm{bp}$ interlaced break caused by the DNA breakage. Moreover, the gap between -1 and +1 nucleotides at both ends of this 4 bp break can be linked to drug molecules.

After years of research, there are many topo-II inhibitors acting on DNA cleavage gates. Quinolones are commonly used antimicrobial agents in clinic at present. Fluoroquinolones enhance absorption and greatly increase the antimicrobial and bactericidal effects. Levofloxacin is one kind of the fluoroquinolones. However, the role of T-DNA and how it is captured and the mechanism through the whole complex are still under study. Perhaps it will become a target for new anticancer and antimicrobial drugs [5].

\section{Aims and Objectives}

Many bacteria are now resistant to drugs and it is important to find new drug sites. My aim is to solve the structure of topoisomerase IV-DNA-drug complex and investigate the link between the closed ATPase domain binds and T-segment of 191bp DNA and the positioning of the G-segment DNA in the G-gate by negative staining electron microscope. 


\section{Materials and Methods}

\subsection{Preparation and purification of the Topoisomerase IV-DNA-Levofloxacin complexes.}

The preconceived DNA is $191 \mathrm{bp}$ has a molecular weight of 126,060 Da, which G-DNA and T-DNA binding domains have been engineered into this synthetic, and ParE as a MW of 72000 Da while ParC has a MW of $92000 \mathrm{Da}$.

An equimolar complex of ParC, ParE and DNA was formed in the 10 micromolar range by mixing the correct combinations of ParC, ParE and DNA 5.38 uM, $71.8 \mathrm{uM}$, 58.3uM respectively.

High performance liquid chromatography was used for the purification of complex. The topoisomerase IVDNA-drug was purified by high performance liquid chromatography (HPLC) using AKTA high performance liquid chromatography system. More purified samples can be selected by observing the trace map.

\subsection{Negative Staining}

The grid covered with a carbon layer was taken to Pelco Easiglow for glow discharge, and the glow discharge time was 60 seconds at the pressure of 0.38 mbar. The purified topoisomerase IV-DNA-levofloxacin sample was then pipetted onto the carbon layer side of the grid. After that, stained with $2 \%$ uranyl acetate. Negative staining stains the background to achieve a good contrast between the black background and the white protein.

\subsection{Electron Microscope of Negative Staining}

Electron microscope was used to collect data for subsequent complex structure studies. The experiment was carried out using a Fei-Tecnai F20 electron microscope located at the Institute of Cancer Research. The stained grid was transferred to the electron microscope at room temperature. With the defocusing of $-3.49 \mathrm{um}$ and the magnification of $50000 \mathrm{x}$, the sample was imaged by a $200-\mathrm{kV}$ electronic gun. And the obtained images were recorded by TIETZ F415 (4K*4K) CCD camera with 1.732 pixels. A total of 55 images of the complex were obtained. Because the proper concentration of the sample for dyeing was not known before, sample were diluted many times until a clearer background can be seen on the computer.

\subsection{Data Processing}

Microscopic images were processed by Reion 3.0. the figure 1 of work flow explains the operation steps of Relion 3.0. Because this experiment was using negative stained data, the contrast transfer function (CTF) estimation, which is helpful to generate phase contrast in the micrographic images, was not needed. After import, there would be a directory created. The next step was manual-picking, which needed to select a large number of proper particles in every micrograph manually. Some of particles in the microscopic image were topoisomerase IV-DNA-drug complex recorded by electron microscopy at different orientations. The selected particles were classified by 2D Classification. 2D Classification is an automatic selection step to classify particles according to different orientations. Choose better and clearer images from 2D Classification as template for Auto-picking, which decide the kinds of particles would be picked and affect the final model at a great extent.

Auto-picking can select more particles. As for the last 2D classification, the selected classes were for the final 3D classification. The reference map for the 3D classification was the structure of Topoisomerase IIDNA-AMPPNP complex from Saccharomyces cerevisiae whose PDB ID is $4 \mathrm{GFH}$ solved by X-RAY diffraction at the resolution of 4.408 A. Finally, UCSF Chimera was used to view the resulting $3 \mathrm{D}$ model.

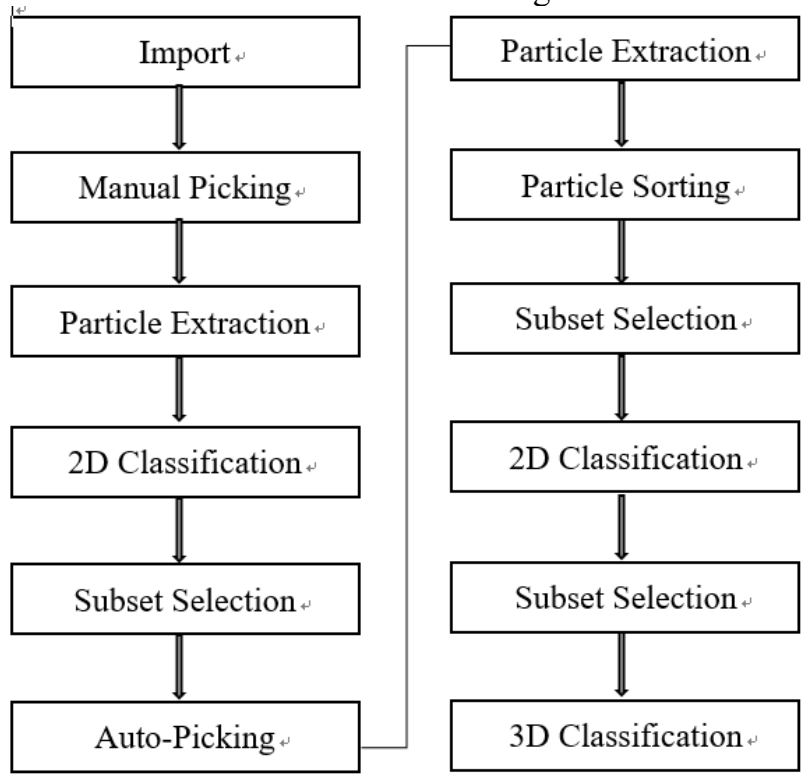

Fig 1. Workflow of the data processing by Relion 3.0.

\section{Results}

These white particles in the green circle are possible and interesting protein in the different orientations (fig2). 1521 particles were picked in the manual-picking from 55 micrographs. Particle diameter was set to be $200 \AA$ and pixel size was $1.732 \AA$ in the manual-picking. Number of classes in the 2D Classfication is dependent on the number of particles picked. There were 15 classes for this process and after comparison, 10 classes were chosen as the template for the auto-picking and 5 classes were discarded.

The resulting 53882 particles were picked in the autopicking. Auto-picking was helpful to select these missed particles by manual-picking. 53882 particles were used for last 2D Classfication for 25 iterations, classified into total 30 classes. But only 6 classes were used for the 3D Classfication containing particles are 587.

Finally, there were 578 particles for the 3D classification after sorting, using the protein model whose PDB ID is 
$4 \mathrm{gfh}$ as the reference map to generate 1 class. It will be better if the resolution could be improved by the further refinement which could select more good particles from the bad classes as mentioned above. But on the other side, there may be no need to have other refinements if the concentration of the protein is low and the number of high-quality micrographs also is not enough.
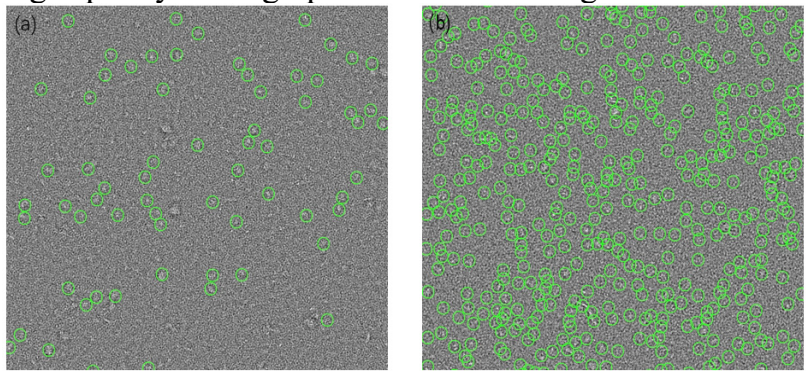

Fig 2. Manual-picking (a) and auto-picking (b). Micrographs from negative stain and the total micrographs are 55 . The diameter of the green cycle is $200 \AA$. The white points are the protein in the different orientations with the pixel size is 1.732

$\AA$.

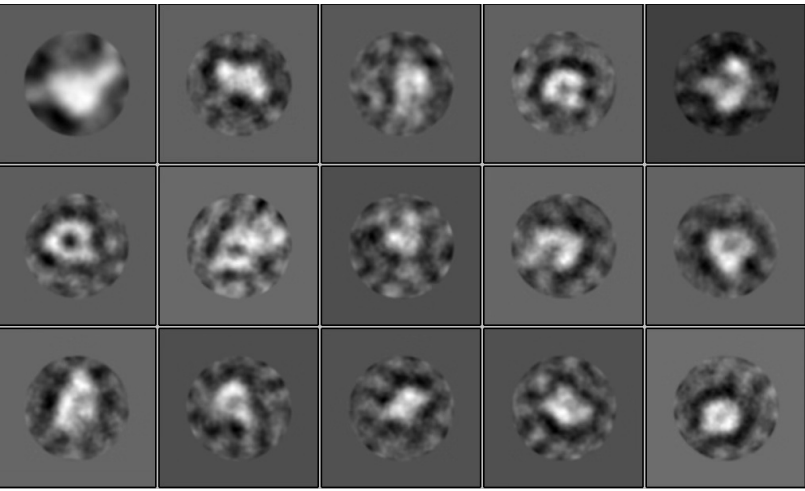

Fig 3. 2D Classification after manual-picking. The selected good classes contain 1521 particles.

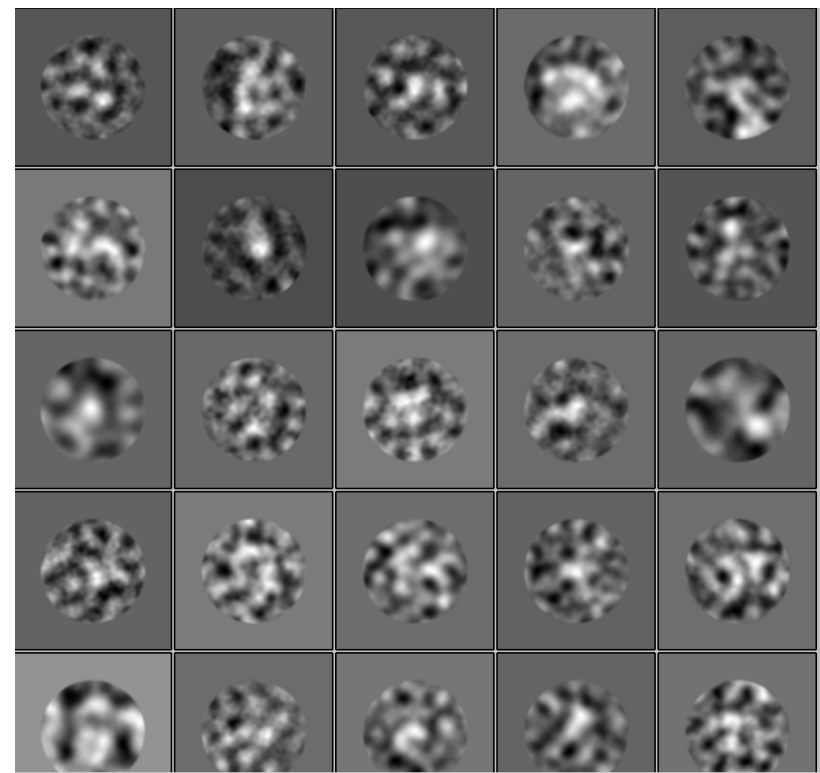

Fig 4. 2D Classification after sort using for the 3D Classification. The background of some of them is not clean containing 'noise'. Only picked 6 of them that obtain 287 particles.

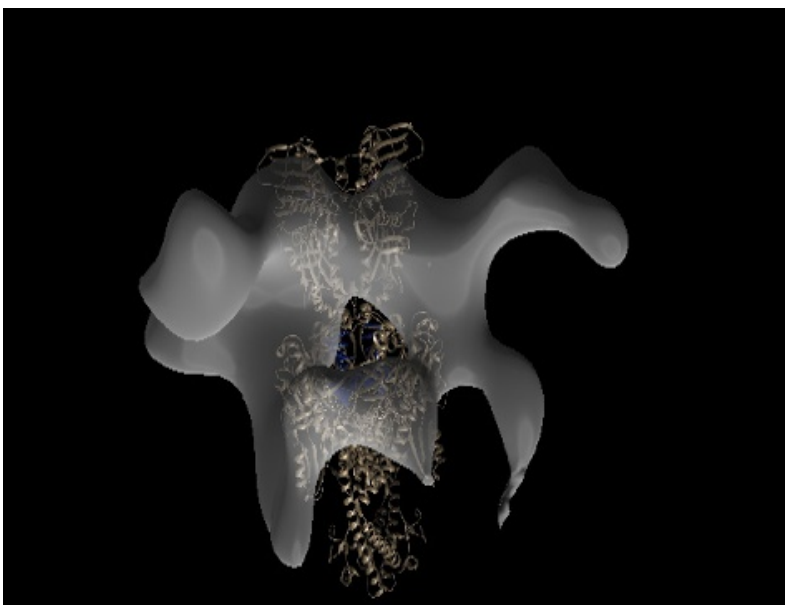

Fig 5. Final 3D structure established by the 55 micrographs from negative staining electron microscopy.

\section{Discussion}

\subsection{Negative Staining}

The negative staining is used to improve the contrast between the protein and the background, so that the complex can be better displayed on the micrographs. the negative staining needs to contain the high concentration of sample and then could provide a better signal-to-noise ration. Most of the 55 microscopic images are filled with white particles, but these particles are not only ideal complexes, but also parts of complexes, ice and various impurities. Therefore, the negative stain could not achieve the high resolution which leads to the final structure of Topo IV-DNA-levofloxacin is a lowresolution model.

\subsection{The analysis of the final model}

The final structure does not show many details such as drug molecules and DNA but the general structure is visible at such a low resolution, which shows ATPase domains of ParE and CTDs (C-terminal domains) of ParC and $\mathrm{N}$-gate as well as $\mathrm{C}$-gate in this model (as shown in the Fig 5).

The expected complex is a $191 \mathrm{pb}$ DNA composed of G-DNA and T-DNA linked to a closed ATPase domain. 14-mer DNA passes through a $22 \AA$ cavity made up of Pare44 ATPase domains. The 14mer DNA of topoisomerase is anamorphic, i.e. there are 5'GCATAT binding sites at both ends of this DNA. The part that is bound to ParE is bent, and the other part in the solution is not bent existing in a $\beta$ form helical structure. This proves that the 5'GCATAT DNA has two configurations, one is the bending configuration in the protein pore and the other is the non-bending configuration of the $\beta$-type helix. At higher resolutions, such as $1.97 \AA$, two short strands of DNA can be seen linking to each side of ParE44 and comparatively speaking, the DNA bound to both sides of ParE44 is more stable than the DNA bound to the hole. When the ATPase domains capture T-DNA, there is a kink causing the DNA to bend 40 degrees. In 
order to stabilize the structure of DNA bound to ParE pore, positively charged residues of arginine and lysine react with the main strand of DNA. What is more, the binding of DNA to protein is asymmetric because DNA asymmetrically binds to residues in the cavity (formed by ATPase domain). And The bottom DNA binds more symmetrically to the top DNA. Mutations in residues affect DNA transmission and ATPase domain activity. Many results have shown that short DNA double strands stimulate ATPase activity. Other short double-stranded DNA binding to the CTD region of ParC or DNA-gate could also stimulate ATPase activity.

In terms of the cycle of mechanism of topoisomerase, ATPase domain closes and T-DNA and G-DNA are linked together, which occurs after capturing T-DNA and ATPase domain closes. Although from this lowresolution image, ATPase domain is open, this structure is indeed a conformation of protein complex structure, which occurs when preparing to capture T-segment. What is more, Fig5 shows part of DNA binds with the TOPRIM domain and longer part of DNA is outside the complex. There are several possible reasons. First of all because negative staining itself belongs to low resolution, it is impossible to distinguish the fine differences from different conformations of protein complexes in the obtained microscopic images. It is likely that the particles of this ATPase domains open conformation dominate in all particles of different conformations. And the second reason is that high-quality micrographs are not enough. Last but not least the final result also shows it possibly because the failure of binding T-DNA with ATPase domain that the final result is not the excepted model because part of DNA can be seen out of the complex.

\section{Conclusion}

From the trace of the purification, the protein has been purified using HPLC and the 56th fraction of the 400 $\mathrm{mM} \mathrm{NaCl}$ was chosen for the negative stain as the sample. There are many factors affecting the result of negative staining, such as the concentration of the sample and PH. Actually, the final result does not agree with the excepted model because of several reasons such as low resolution, the unexpected dominant conformation and the failure of the DNA binding and so on.

The expected structure of topoisomerase IV binding with the 191bp DNA that contains G-DNA and T-DNA binding together has never been observed before. We would like to see the linkage between the T-segment and the closed ATPase domain. The T-DNA binds with the 14 mer DNA in the cavity caused by the closed ATPase clamp and 6 bp DNA also binds with the both sides of the TOPRIM domain of ParE. The short DNA binding with the residues of the cavity could affect the activity of the ATPase meanwhile the mutations of the residues decrease the activity of the topoisomerase IV at a great extent.

Although many topoisomerase inhibitors have been found and used in the treatment of diseases, the formation and reversal of topoisomerase complexes remain unclear. However, in recent years, with the widespread use of quinolones in clinical practice, bacterial resistance to quinolones has increased, which poses a serious threat to clinical treatment. Therefore, finding a new way to inhibit the activity of topoisomerase IV is very urgent. The study of ATPase domain binding with the DNA can provide a new view for the different drug site. It can also help understand the mechanism of the moving of T-DNA and the interaction between protein and DNA.

\section{References}

1. Huang, X. and Chen, X., 1990. Supercondensed structure of plasmid pBR322 DNA in an Escherichia coli DNA topoisomerase II mutant. Journal of molecular biology, 216(2), pp.195-199.

2. Neuman, K.C., 2010. Single-molecule measurements of DNA topology and topoisomerases. Journal of Biological Chemistry, 285(25), pp.18967-18971

3. Muzammil, E.M., Khan, A. and Stuparu, M.C., 2017. Post-polymerization modification reactions of poly (glycidyl methacrylate) s. RSC Advances, 7(88), pp.55874-55884.

4. McClendon, A.K. and Osheroff, N., 2007. DNA topoisomerase II, genotoxicity, and cancer. Mutation Research/Fundamental and Molecular Mechanisms of Mutagenesis, 623(1-2), pp.83-97.

5. Laponogov, I., Pan, X.S., Veselkov, D.A., Skamrova, G.B., Umrekar, T.R., Fisher, L.M. and Sanderson, M.R., 2018. Trapping of the transportsegment DNA by the ATPase domains of a type II topoisomerase. Nature communications, 9(1), p. 2579 . 